\title{
Prospects for the gene manipulation of Streptococcus bovis
}

\author{
P Javorsky, I Vanat, P Pristas, M Pravdova, I Styriak, V Kmet \\ Institute of Animal Physiology, Slovak Academy of Sciences, \\ Soltésovej 4-6, 04001 Kosice, Slovakia
}

In general, the realisation of goals to control the digestive processes of ruminants via genetic manipulation of rumen bacteria is limited by the lack of information about the genomes of important rumen bacteria, cloning systems, stability of cloned DNA in rumen bacteria and on factors affecting the whole rumen ecosystem.

Information about the genome of $S$. bovis is restricted to a cloning study of genes in the lactose catabolic pathways by cloning of aamylase genes and by some details in their cryptic plasmids. The bacteriophages of $S$. bovis occurring in the rumen have potential for use as a vectors. In our laboratory five $S$. bovis bacteriophages have been isolated and partly characterized. Restriction endonucleases profiles of phage DNA were completely different for the five isolates. A detailed characterization of the phage genomes could allow their use as vectors in the future. Despite litlle use of naturally occurred plasmids and phages of $S$. bovis as vectors to date, some shuttle vectors, capable of replication in Gram positive bacteria and easier experimental subjects such as $E$. coli, have been used for transformation of $S$. bovis by conjugation, electroporation and protoplast transformation. In an endeavour to develop a suitable transformation system, we have transformed $S$. bovis AO 24/85 with plasmid pNZ12 by electroporation with transformation efficiency $1.1 \times 10^{3} / \mu \mathrm{g}$ DNA.

Recently, Whitehead and Flint (1995,
FEMS Microbiol Lett, 126(2), 165-169) described the heterologous expression of an endoglucanase gene (endA) from the $R$. flavefaciens 17 in S. bovis. This is the first gene transfer between two different rumen bacteria.

The rumen is a complex ecosystem with many different microorganisms and with a high potential for natural transfer of genetic material. To protect their individual genetic identities various restriction-modification systems can be expected within the rumen microorganisms. Recently restriction endonucleases have been isolated and characterized from ruminal bacteria. In our laboratory more $S$. bovis strains were tested for their restriction activities. We have developed a very simple, rapid and effective method for testing $R E$ activity of rumen bacteria. By this method we detected, isolated and characterized the restriction endonuclease Sbvl, and isoschizomer of Haelll, from rumen amylolytic bacterium $S$. bovis II/1. Enzyme Sbvl

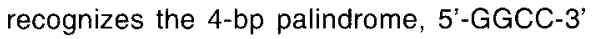
and cleaves DNA after the second $G$ in the sequence, producing blunt ends.

Further study of restriction-modification systems of selected ruminal bacterial species has significant implications for genetic manipulation of these bacteria, especially for the introduction, expression and stability of foreign DNA within the rumen bacteria and the whole rumen ecosystem. 